\title{
U m sistema difuso inteligente para avaliar informações de usuários na Internet
}

\author{
A ntonio C esar Ferreira G uimarães \\ Inteligência A rtificial - Sistemas Computacionais Inteligentes. \\ Divisão de Reatores, Instituto de Engenharia $\mathrm{N}$ uclear. \\ tony@ien.gov.br
}

\begin{abstract}
Resumo
Este trabalho consta do desenvolvimento de um Sistema Inteligente na Internet (SII) para indicar o nível de aquisição material dos visitantes em páginas na WWW (World Wide Web). A partir do Tempo de permanência (Tp), da Freqüência de visitas (Fv) e de um Índice (IDLI) que mede o interesse do usuário na página Web, o sistema estabelece um padrão existente nestas informações relacionadas com o número de Ordens de Compras dadas por um usuário. Desta forma, as variáveis tratadas como pares de entradas/saídas estabelecem um sistema adaptativo difuso que possui a capacidade de aprender com o tempo e a generalizar para definir novos padrões de usuários. Uma aplicação da metodologia proposta foi apresentada para efeito de ilustração. Com os resultados obtidos, pode-se definir um número de ordem de compra para cada item oferecido na página Web, estabelecer limites para o estoque adequado, ou estabelecer critérios de exibição de ofertas de produtos na página Web da empresa.
\end{abstract}

Palavras-chave

Lógica difusa; Internet; Anfis.

\section{Fuzzy intelligent system to evaluate user information on Internet}

\begin{abstract}
This work consists of the development of an Intelligent System in the Internet (ISI) to indicate the level of the visitors' material acquisition in pages in WWW (World Wide Web). Starting from the Time of permanence (Tp), of the Frequency of visits (Fv) and of a Index (IDLI) that measures the interest of the users in the page web, the system establishes an existent pattern in these information related with the number of Orders of Purchases given by a user. In this way, the variables as input/output pairs establish an Adaptive Neural Fuzzy Inference System (Anfis) that possesses the capacity to learn with the time and to generalize to define new patterns of users. For illustration effect an application of the methodology proposal was presented. With the obtained results, can be defined a number of purchase order for each item offered in the page web, to establish limits for the appropriate stock, or to establish approaches of exhibition of offers of products in the page web of the company.
\end{abstract}

Keywords

Fuzzy logic; Internet; Anfis.

\author{
INTRO DU ÇÃO
}

U ma das maiores preocupações deempresas comerciais ligadas à Internet ésaber exatamente quais informações mostrar ao usuário e desta forma manter sua atenção em ofertas ou produtos em geral, impedindo que o mesmo não abandonea página ( home page) Web, ou seja, mantenha-se navegando no siteo maior tempo possível. As informações devem ser interessantes, seja no visual ou nas ofertas eprodutos oferecidos, deforma queo usuário deseje voltar o maior número de vezes à páginaWeb eque o interval o entre uma visita e outra seja em um intervalo detempo cadavez menor. V árias empresas comerciais queutilizam o sistemade comércio eletrônico ( shopping) fazem detudo para atrair a atenção dosusuários. Basicamente, grande número deempresas usa o recurso de um cadastro, no qual o usuário, após o seu preenchimento, passa a dispor de um nome-de-usuário (user name) e de umasenha ( password). A partir daí, as empresas identificam o clientee o seu retorno, edestaforma oferecem-se informações personal izadas sobre produtos para novas aquisições, como, por exemplo, alivrariaA mazon, acessada por meio daU RL http:// www.amazon.com, em que novos livros com características semel hantes são ofertados após a confirmação da sua identificação. 0 s livros que possuem palavras ou contexto semelhantes são oferecidos em sua página personal izada.

Existem outras configurações de políticas de amostragem de ofertas, como é o caso, por exemplo, da L ojas Americanas, acessada através da URL http:// www.lojasamericanas.com, na qual a atual ização éfeita assim que o usuário abre uma nova página. A s ofertas constantes na página principal são atual izadas instantânea eal eatoriamente, com produtos misturados devárias naturezas, to da vez que uma nova página éaberta. U tilizando o ícone deatualização na barra de ferramentas do navegador, novas ofertas são também disponibilizadas al eatoriamente, sem nenhum critério. $U$ tilizando o recurso de abrir uma nova janela pelo navegador, a página da L ojas A mericanas também apresenta novas informações, se comparadas com aquel as apresentadas em uma página previamente aberta. Esta técnica utiliza recursos de programação apenas e não possui um sistema inteligente que possa filtrar as informações que 


\section{A ntonio C esar Ferreira G uimarães}

deverão ser disponibilizadas para o usuário em particular, como no caso da A mazon. A diferença entre o quea A mazon oferece de conteúdo e uma página que utilize um sistema inteligente é que a A mazon não possui a preocupação de verificar o tempo em queo usuário permaneceem um item de pesquisa eo potencial de compra através de um indicador de interesse (ILIP - I nterest L evel I ndicating Point) oferecendo apenas mercadorias para compra similares baseados em alguma comprafeita ou pesquisarealizada.

F oi proposto em I p et al. (2000) o desenvolvimento de um sistema( I ntelligent I nternet I nformation D elivery System - IIID S) caracterizado por suacapacidadede "máquina de aprendizado", baseado nos dados de movimentos pontuais da páginaWeb gerados pelos usuários ea partir deste ponto avaliar as preferências, os locais mais visitados erelevantes por meio dos princípios delógicadifusa (F uzzy L ogic). N estecaso, ametodologia propostasugere autilização deum sistema inteligenteetomando o exemplo daA mazon se assemelhariaaumasituação em que os recursos da A mazon oferecidos aos usuários viessem a oferecer informações personal izadas com as ofertas que mais apontam nesta direção, de acordo com as conclusões feitas em I p et al. (2000).

A pesar da forma inteligente proposta, sempre existe a necessidade dese identificar o usuário para oferecer um conteúdo "personalizado em potencial", baseado em informações geralmentenão consideradas em muitas páginas daWeb.

A propostafeitanestetrabalho éuma contribuição ao já desenvolvido (I p et al., 2000) ese preocupaem apresentar conteúdo atualizado na página sem a necessidade de identificação do usuário. M uitos usuários, durante as visitas, não se identificam e nem fazem cadastro para suas consultas. A partir de informações, tais como tempo de permanência, freqüência de visitas à página e o ID LI, deve-se desenvolver um sistema que modele a coleção de dados de entrada/ saída, proporcionando adaptação ao sistema e fornecendo a habilidade de aprender o relacionamento embutido na coleção de dados de entrada/ saída.

$\mathrm{N}$ o item 2, será descrita a metodologia envolvida para definição do sistema de inferência difusa neuroadaptativa (A nfis-A daptiveN eural F uzzy I nferenceSystem). N o item 3, será apresentado um caso exemplo para demonstrar a metodologia proposta. $\mathrm{N}$ o item 4 , os resultados foram apresentados ediscutidos para estecaso exemplo. $\mathrm{N}$ o item 5, serão relatadas as conclusões finais sobreo trabalho.

\section{MET O DO LO GIA PRO PO ST A}

U m sistemadeinferênciadifuso neuroadaptativo ( A nfisAdaptiveN eural F uzzy I nferenceSystem) éum sistemade inferência difuso (FIS - F uzzy I nferenceSystem) quepode ser treinado com um al goritmo deretropropagação para modelar um conjunto de dados entrada/ saída (U hrig et al., 1997). 0 FIS, permitindo adaptação, contempla o sistema difuso com ahabilidade deaprender inter-relações embutidas nas entradas esaídas dos dados utilizados.

A estrutura de rede da A nfisfacilita o processo decomputação do vetor gradiente que relaciona a redução de umafunção erro parauma modificação nos parâmetros de umaFIS. A ssim queo vetor gradienteé obtido, um número derotinas deotimização podeser aplicado para reduzir o erro entre a saída real e a obtida (U hrig etal., 1997). N a literatura de redes neurais, isto é conhecido como aprender com o exemplo.

0 modelo de A nfis utilizado neste trabalho é do tipo Sugeno (conhecido como TSK fuzzy model), proposto por Tagaki \& Sugeno (1985) eSugeno \& K ang (1988). Takagi \& Sugeno (1985) propuseram utilizar as regras IF-THEN daseguinteforma:

$$
\begin{gathered}
\mathrm{L}^{(l)}: \mathbf{I F} x_{1} \text { is } F_{1}^{l} \text { and } \cdots \text { and } x_{n} \text { is } F_{n}^{l} \\
\text { THEN } y^{J}=c_{0}^{\prime}+c_{1}^{\prime} x^{\prime}+\cdots+c_{n}^{\prime} x_{n}
\end{gathered}
$$

$O$ nde, $F$ ' são conjuntos difusos, $c_{i}$ são variáveis reais, $y$, é o valor desaída dos sistemas devido à regra $L^{(1)}, \mathrm{el}=1,2$, ..., M . O u seja, a parte relacionadal F édifusa, mas a parte T H EN éreal ( crisp) - a saída é uma combinação linear das variáveis deentrada( input). Paravariáveis deentrada reais cujo vetor $\mathrm{x}=\left(\mathrm{x}_{1}, \ldots, \mathrm{x}_{\mathrm{n}}\right)^{\top}$, a saída éy $(\mathrm{x})$ o sistema difuso do tipo Takagi eSugeno eumamédiados pesos dos valores dey's:

$$
\mathrm{Y}(\mathrm{x})=\left(\sum_{l=1}^{M} w^{l} \cdot y^{l}\right) /\left(\sum_{i=1}^{M l} w^{l}\right)
$$

O nde os pesos $w^{\prime}$ implicam o valor verdade da premissa da regra L (I) para as variáveis de entrada ecalculadacomo:

$$
\mathrm{W}^{l}=\prod_{i-1}^{n} \mu_{F_{l}^{\prime}}\left(x_{i}\right)
$$




\section{U m sistema difuso inteligente para avaliar informações de usuários na Internet}

Mais detalhes sobre TSK fuzzy model podem ser encontradosem (Wang, 1993). A configuração do sistema difuso do tipo Takagi \& Sugeno émostradanafigural.

0 toolbox do (Matlab, 2000), foi empregado para representação do problema.

\section{FIGURA 1}

\section{C onfiguração básica do sistema difuso do ti po Takagi \& Sugeno.}

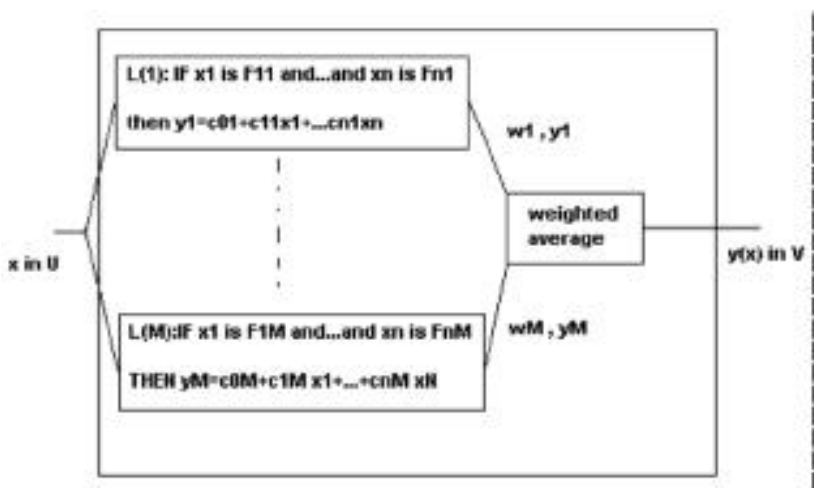

\section{APLICAÇÃO DA METO DOLO GIA}

Para aplicação da metodologia proposta aqui, as informações e dados de navegação de usuários utilizados em (I p et al., 2000) foram também empregadas nesta aplicação. Serão descritas a seguir algumas etapas de desenvolvimento neste referido trabal ho. Foram obtidas inicialmente informações extraídas para três visitantes da página Web de um empresa virtual comercial (veja Table 1 em I p, 2000). Estes dados foram compilados automaticamente e gravados por meio de um contador utilizando alinguagem de programação em V isual B asic Script (VBScript). M aiores informações sobreV BScript podem ser obtidas por meio daU RL daM icrosoft http:/ / www.microsoft.com. Três conjuntos dedados estatísticos são extraídos da informação gravada devido ànavegação dos usuários. D ados estatísticos, tais como, o tempo total de permanência em cada local, Ts, a freqüência de visita de cada local, Fv, e o tempo ocorrido entre duas visitas sucessivas, Ti, foram retirados da tabela inicial e apresentados em uma segunda tabela para calcular os valores do ILIP (vejaT able2 em I p, 2000). U m valor ILIP (Interest L evel I ndicating Point) para cadalocal visitado é determinado (vejaT able $5 \mathrm{em} \mathrm{I} \mathrm{p,} \mathrm{2000).} 0$ s locais com valores ILIP relativamente altos significam que 0 visitante tem maior interesse no local. 0 responsável pelo projeto da página (Web master) deverácolocar mais informações sobreestes produtos ereservar al gum espaço na página principal contendo promoções destes produtos para as próximas visitas do mesmo visitante. É muito possível queainformação em tempo real possaser obtida conforme o desejado, fornecida por um sistema difuso trabalhando em conjunto com a páginaWeb. 0 sautores (I p et al., 2000) declaram que o sistema proposto possui a capacidade de decisão humana relacionada para as vendas de produtos por meio da I nternet sob o conceito da entrega de informação selecionadae, desta forma, tem mais sucesso do quea estratégiadeentrega deinformação intensivatradicional.

O s valores dos I LI Ps determinados demonstraram que nenhum valor deI LIP podeser determinado se o usuário tem apenas uma visita em um local da página em um período de navegação e, também, quelocais com o tempo de permanência grande e visitado em uma única navegação, um I LIP maior éobtido. 0 utraobservação é que deslocamentos entre locais de pesquisa na página podem ser sempreidentificados.

E m outra tabela (vejaTable6, I p et al., 2000), apresentada aqui em forma de figura, figura 2, foram compilados para cada visitante o Ts, o F v, os valores calculados I LI P e o número de ordens de compra ( $\mathrm{N}$ o. of order) feitas em cada local da página visitada para um período de observação de 30 dias.

Baseado nestes dados da figura2, foram definidos então os pares de entrada/ saída para o exemplo da aplicação da metodologia A nfis proposta aqui, conforme descrito a seguir.

C ada visitantefoi selecionado como um tipo de conjunto dedados, ou seja, o visitante ( Visit 1) denúmero 1 foi o conjunto de treinamento (training). 0 visitante de número 2(Visit2) foi o conjunto deverificação (checking), e o visitante de número 3 ( $V$ isit 3 ), o conjunto de teste (testing). 0 s dados foram assim considerados parafacilitar a implementação da proposta enão impedem no futuro deseutilizarem dados mais específicos. 


\section{A ntonio C esar Ferreira G uimarães}

O nde,

$\mathrm{BE}$ - B usinessE quipment, $\mathrm{C}$ om - C omputer, F/W - F ood/wine, ID - I nterior D esign, SM ShoppingM all, eTele-Telecomunication.

O s valores deTs, Fv el LIP são os dados de entrada, e os valores de 0 rdem são os dados de saída.

Em seguidaà preparação dos dados, a FIS ( fuzzy inferencesystem) écriada, usando o tool box (M atlab, 2000). A lgumas propriedades da FIS Sugeno desenvolvidas contendo o método de implicação eoutras são apresentadas:

nome: 'Produto'

type: 'sugeno'

andM ethod: 'prod'

orM ethod: 'probor'

defuzzM ethod: 'wtaver'

impM ethod: 'min'

aggM ethod: 'max'

input: [ $1 \times 3$ struct]

output: [ $1 \times 1$ struct]

rule: [ $1 \times 27$ struct]

N o próximo item, serão apresentados os resultados exibidos em forma gráfica.

\section{RESULTADOS}

$\mathrm{N}$ afigura 3, os valores calculados de ID LI em (I p et al., 2000), queforam exibidos na figura2, são novamente apresentados em forma gráfica juntamentecom os valores estimados com o FIS Sugeno gerado nesteestudo, para efeito de comparação.

O s gráficos relacionados a v1c, v2c, ev3c são referentes aos valores cal culados. O s gráficos v1e, v2ee v3e são os relacionados aos estimados pela metodologia $A$ nfis aqui proposta. $\mathrm{N}$ afigura 3, o eixo dos $x$ representa os locais visitados na páginaWeb, e o eixo y, os valores relativos ao número de ordem feita paraum determinado produto e para um específico usuário. Paraeste exemplo, os locais visitados são em número de 7, conforme descrito anteriormente e relacionado na parteinferior dafigura 3.

FIGURA 2

FIGURA 3

\section{$D$ ados dos três usuários}

\begin{tabular}{|c|c|c|c|c|c|c|c|c|c|c|c|c|}
\hline & & Visit 3 & & & & Shooging & Visit 1 & & & & Visit 2 & \\
\hline IILIP & Ordem & Ts(min) & $F_{v}$ & ILIP & Ordem & & Ts(min) & Fv & ILIP & Ordem & Ts(min) & Fv \\
\hline 86.1 & 1 & 23 & 3 & 7.5 & 0 & $\mathrm{BE}$ & 522 & 15 & 85.5 & 2 & 210 & 10 \\
\hline 10.1 & 0 & 724 & 21 & 95.7 & 3 & Com & 270 & 10 & 77.9 & 1 & 15 & 2 \\
\hline 91.6 & 3 & 768 & 20 & 94.3 & 4 & $F / W$ & 103 & 11 & 57.2 & 1 & 407 & 15 \\
\hline 37.4 & 0 & 110 & 4 & 19.2 & 0 & ID & 440 & 19 & 87.7 & 2 & 78 & 6 \\
\hline 60.5 & 0 & 542 & 15 & 81.8 & 2 & Off & 653 & 18 & 92.3 & 3 & 115 & 8 \\
\hline 89.7 & 3 & 209 & 12 & 44.4 & 1 & StA & 221 & 8 & 56.1 & 0 & 388 & 13 \\
\hline 60.2 & 1 & 441 & 17 & 76.8 & 2 & Tele & 78 & 5 & 22.3 & 0 & 147 & 7 \\
\hline
\end{tabular}

D ados comparados entre o calculado e o estimado com o método A nfis

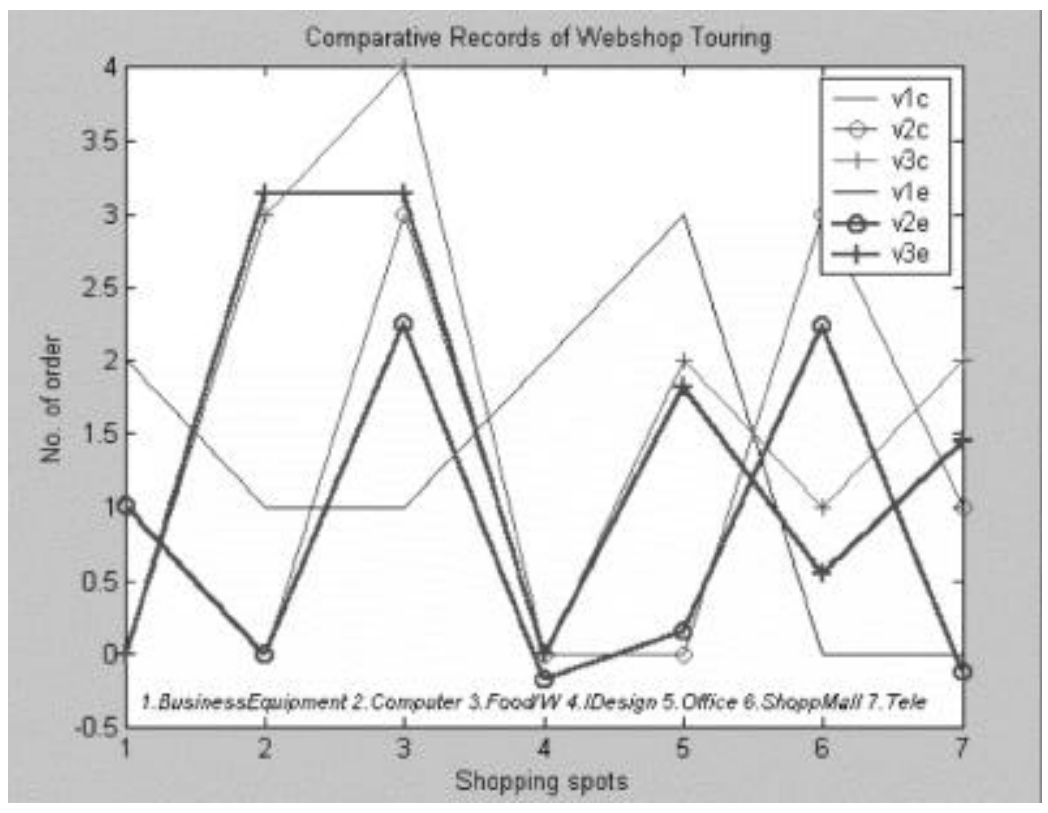

0 primeiro conjunto de dados detreinamento éo v1c, utilizado paradesenvolver a FISSugeno, juntamentecom o conjunto de dados v2c, utilizado como conjunto de verificação, cujafinalidadeéa dehabilitar o sistema difuso ageneralizar. 0 conjunto de dados v3c foi utilizado como conjunto de teste, cujafinalidade é avaliar a capacidade de o sistema difuso reconhecer algum conjunto novo apresentado ao FIS. É importanteressaltar queesteconjunto v3c não foi utilizado anteriormente para geração daFIS. N o primeiro caso (v1c), não se verifica diferença entre o cal culado eo estimado. $\mathrm{N}$ o segundo caso (V2c), a estimativa podeser consideradaboa. E no terceiro caso (v3c), a estimativa a partir de um conjunto novo pode ser também consideradaboa. 


\section{U m sistema difuso inteligente para avaliar informações de usuários na Internet}

Com relação à aquisição de informações de navegação dos usuários, além do citado anteriormente com a linguagem de programação VBScript daM icrosoft, existem dois ambientes computacionais, Webalizer (2002) e o WebTrends (2002) , para monitoração do arquivo "log". Este arquivo armazena informações sobre a navegação do usuário durante uma pesquisa na páginaWeb eé encontrado no servidorWE B da empresavirtual.

\section{CONCLUSÕES}

Estetrabal ho apresentou uma proposta inteligentepara exibir informações selecionadas nal nternet. Para tal, um sistema de inferência difuso, FIS ( F uzzy I nference System), foi desenvolvido utilizando ametodologiaA nfis (AdaptiveN eural F uzzy I nferenceSystem) do tipo Sugeno $\&$ Tagaki.

Com o "sistema" proposto, pode-se estimar o nível de interessee potencial de comprade um usuário. 0 autor acredita queo sistema difuso acoplado à páginaWeb possa ser utilizado em tempo real para auxiliar a apresentação das informações sobre produtos oferecidos pela empresa virtual, sem a necessi dade de identificação do usuário e de maneira mais inteligente e criteriosa.

0 arquivo "log" daempresa deveráser utilizado para se extraírem estatísticas para um período de tempo para definição deum padrão. A partir daí, surgirão novos dados para serem utilizados na generalização daFIS inicialmente produzida, em um processo contínuo de atualização do padrão.

Este tipo de funcionamento éuma maneira mais inteligentee mais comercial, se comparado aos dois exemplos citados anteriormente no texto do trabal ho, A mazon e L ojas A mericanas, e acredita-se que ocorra em muitas outras empresas comerciais virtuais.

Com a metodologia proposta aqui, uma empresa comercial virtual estaria livre, após identificar o usuário, de coletar informações relevantes queserão disponibilizadas quando do retorno do usuário, se ele voltar, como éo caso daA mazon e, além disso, não estaria preenchendo a página principal com ofertas indesejáveis, sem a necessidade de identificação do usuário, enão se preocupando seo usuário vai voltar ou não ao siteda página, como éo caso da empresa L ojas A mericanas.
O s dados aqui utilizados foram obtidos do exemplo proposto em (I pet al., 2000) edevem ser considerados apenas importantes do ponto de vista de demonstração. U m conjunto maior de dados deveser selecionado, deforma a permitir ajustes no sistema $A$ nfis durantea sua fase de treinamento, teste e validação. É importante ressaltar que o toolbox, pela suafacilidade de implementação, foi utilizado apenas como um simulador. 0 sistemapodeser total mente desenvolvido em uma linguagem apropriada.

Ferramentas automatizadas podem ser empregadas para análise delog. Scripts podem ser desenvolvidos para extração de informações do log, para em seguida serem incorporados no sistema difuso que vai estimar os níveis de interesse, para em seguida serem repassados à programação Web, quedisponibilizaráinformações selecionadas para o usuário.

A rtigo aceito para publicação em 22-11-2002

\section{REFERÊNCIAS}

Ip, R. W. L., H .C.W. Lau, \& F.T. Chan 2000, A n intelligent Internet information delivery system to evaluate site preferences: expert systems with applications. [S. I.] : Pergamon, 2000. p. 18, 33-42.

MANUAL do fuzzy toolbox do matlab 6. [S. I. : s. n.], 2000.

SU GEN O , M .; KA N G , G. T. Structure identification of fuzzy models. Fuzzy Sets Syst., v. 28, n. 15, 1988.

TAKA GI , T.,; SU GEN O , M . Fuzzy identification of systems and Its applications to modeling and control. IEEE Trans. Syst. M an, C ybern., v. 15, n. 1, p. 116-132, 1985.

UHRIG, R.E. et al. Signal validation using an adaptive neural fuzzy inference system. N uclear Technology, v. 119, A ug. 1997.

WAN G, Li-Xin. A daptative fuzzy sustems and control : design and stability analysis. Berkeley : U niversity of C alifornia, 1993.

Webalize. D isponível em: < ttp:// www.mrunix.net/ webalier > . A cesso em: 2002.

WebT rends. < http:// www.netiq.com/webtrends/default.asp> . A cesso em: 2002. 